\title{
A Novel Complication Following Retrograde Intrarenal Surgery: Retroperitoneal Hematoma
}

\author{
Berat Cem Ozgur ${ }^{1}$ and Musa Ekici²
}

\begin{abstract}
Retrograde intrarenal surgery (RIRS) is an efficient method for kidney stones with low complication rates. Reported here is an unusual complication of this surgery; a retroperitoneal hematoma $8 \times 7.5 \times 5 \mathrm{~cm}$ in dimension in a 40 -year female, which was completely resolved by conservative treatment. This is the first reported case of retroperitoneal hematoma following RIRS in the literature. Although this technique is safe, feasible and minimally invasive for kidney stones, urologists should be vigilant for such a complication.
\end{abstract}

Key Words: Retrograde intrarenal surgery, Hematoma, Retroperitoneal.

\section{INTRODUCTION}

Retrograde intrarenal surgery (RIRS) is an option used for the treatment of kidney stones. It has become an efficient and safe method in the treatment of kidney stones of various sizes over time. ${ }^{1}$ Furthermore, RIRS is a less invasive method compared to the other treatment modalities (percutaneous nephrolithotomy and open surgery) and has increased success rate. Although, it is a minimally invasive procedure, there are some complications associated with this method. ${ }^{2}$

Herein, we present a case of retroperitoneal hematoma, which had a rare, but serious complication following RIRS.

\section{CASE REPORT}

A 40-year lady patient was referred to the urology outpatient clinic with the complaint of right-sided lumber pain. She underwent non-contrast enhanced computed tomography (NCCT) and was diagnosed with a kidney calculus of $20 \times 15 \mathrm{~mm}$ size, extending from the right renal pelvis to the lower pole (Figure 1). The patient was scheduled for RIRS for the treatment of the stone.

The operation was performed under general anesthesia and in the lithotomy position. A guidewire (0.038-inch hydrophilic material coated flexible tip guidewire, CookMedical, Limerick, Ireland) was passed to the kidney and its location was confirmed through fluoroscopy (Ziehm 8000, FL, USA). An uretheral access sheath (UAS) (9.5Fr, Cook Medical, Bloomington, USA) was inserted through fluoroscopy via the guidewire. The

1 Department of Urology, Ankara Research and Training Hospital Urology Clinic, Ankara, Turkey

2 Department of Urology, Çorum Hitit University, Çorum, Turkey

Correspondence: Dr. Berat Cem Ozgur, Department of Urology, Ankara Research and Training Hospital Urology Clinic, Ankara, Turkey

E-mail: bcemozgur@hotmail.com

Received: June 25, 2018; Accepted: September 18, 2018 laser probe was sent through a flexible renoscope (Storz Flex-X2, Tuttlingen, Germany) and the stone was broken with Sphinx 30 Litho, Holmium-YAG laser, with pulse energy of $0.5-4.0 \mathrm{~J}$, at a frequency single $4-20 \mathrm{~Hz}$, pulse peak power $15 \mathrm{~kW}$ and 270 micron laser fiber. When image clarity was disrupted, a pumped irrigation set (irrigation $Y$ set) was used to provide pressurized fluid intermittently. A $4.7 \mathrm{Fr}$ double-j catheter of $26 \mathrm{~cm}$ was inserted. Intraoperatively, a single mucosal tear (nearly one $\mathrm{mm}$ ) of lower renal calyx due to laser effect was observed and noted as a minor intraoperative complication. The operation lasted for 70 minutes.

Kidney ureter bladder (KUB) graphy was ordered at the first postoperative day and the DJ catheter was seen to be in place. In the physical examination, no abdominal distension was observed, but there was tenderness. She had pain in the right lower quadrant and flank region. Temperature of the patient was $37.1^{\circ} \mathrm{C}$. Blood pressure was $110 / 70 \mathrm{mmHg}$. Hemoglobin value was preoperative $11.7 \mathrm{~g} / \mathrm{dL}$ and postoperative $11.2 \mathrm{~g} / \mathrm{dL}$. Creatinine level was measured as preoperative $0.6 \mathrm{mg} / \mathrm{dL}$ and postoperative $1.1 \mathrm{mg} / \mathrm{dL}$.

A contrast computed tomography (CCT) was planned and in order to minimize further renal dysfunction, continuous intravenous hydration with isotonic $\mathrm{NaCl}$ at a rate of $0.5 \mathrm{ml} / \mathrm{kg} / \mathrm{h}$ was administered 6 hours before contrast exposure and till 6 hours after the procedure. Also a nonionic contrast agent was used. Based on the CCT, the patient was diagnosed with hematoma of $80 \times 75 \times 50 \mathrm{~mm}\left(10 \mathrm{~cm}^{3}\right)$ in dimension on the second postoperative day. The hematoma started from the level of middle pole of the kidney, surrounding the kidney and extending to the pelvic area $(4 \mathrm{~cm}$ below the lower pole of the kidney) in the retroperitoneal region (Figure 2).

The patient was put on intravenous fluid, antibiotics, (continued with seven-day course of oral ciprofloxacin) and anti-inflammatory treatment. Fever, pulse, blood pressure, and hemogram of the patient were monitored. Subsequently, there was no decrease in hemoglobin 


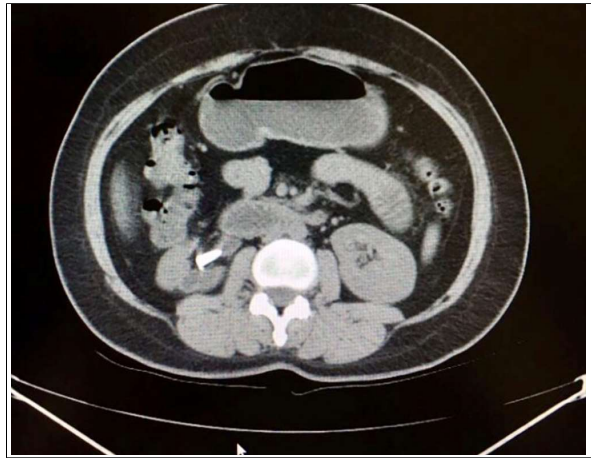

Figure 1: Image of perirenal region in preoperative period.

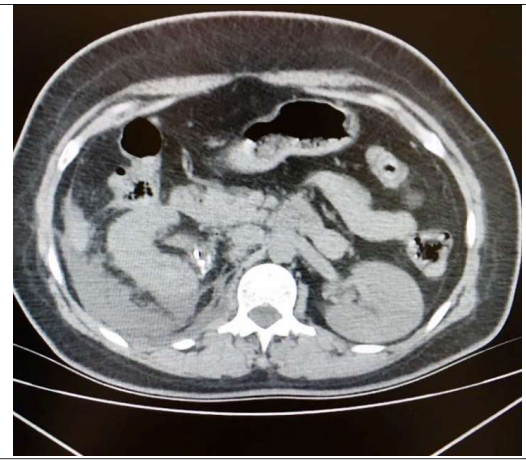

Figure 2: Image of perirenal region in early postoperative period (second day).

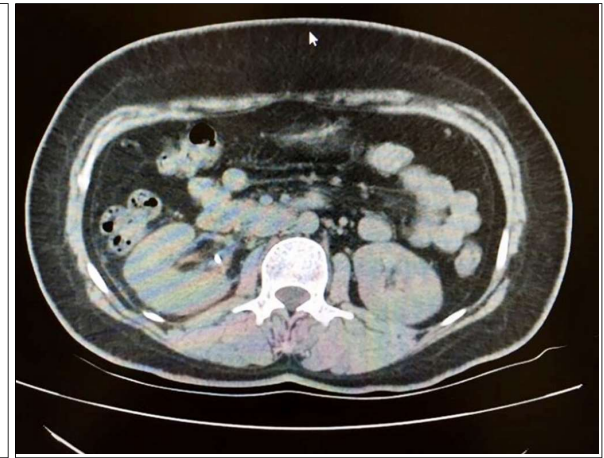

Figure 3: Image of perirenal region on postoperative 3 weeks. level, and no fever; while her pain decreased and disappeared. On the NCCT ordered on the postoperative day 7, hematoma was observed to be resorbed. On the postoperative day 21 , hemoglobin was $12 \mathrm{~g} / \mathrm{dL}$, and creatinine $0.8 \mathrm{mg} / \mathrm{dL}$. NCCT demonstrated completely resolved hematoma (Figure 3 ). She remained asymptomatic at 5 months of follow-up.

\section{DISCUSSION}

RIRS is recommended for the treatment of kidney stones less than $2 \mathrm{~cm}$ in the European Urology Guidelines. ${ }^{1}$ The use of this efficient and less invasive method has become widespread, while complications have been reported with this common use.

The complications seen in RIRS can be classified as intraoperative, early postoperative, and late postoperative. Intraoperative complications include, mucosal injury, perforation and damage to the flexible renoscope, while early postoperative complications include fever, urinary tract infections, stent migration, stone tract, sepsis, retroperitoneal abscess, and late postoperative complications include urethral stricture. 3

Besides these complications, hemorrhage may be seen due to ureteral laseration during insertion of UAS, laser injury or calyceal avulsion. In addition, it may also be caused by parenchymal and forniceal rupture due to a high intrarenal pressure.4,5 The incidence of subcapsular hematoma after RIRS is less than $1 \% .6$ It may spontaneously occur in patients aged over 70 years who are using anticoagulant drugs and have concomitant renal failure. ${ }^{7}$ But such a retroperitoneal hematoma is an exception and is a clinical problem with high rates of morbidity and mortality. It requires a serious management with transfusion and intermittently performed radiological procedures. ${ }^{8}$ There are a few case reports in the literature on hematoma seen following RIRS, but those cases were treated by secondary manipulations. 9,10 Since hematoma transformed into abscess formation in the late period in these cases, open surgical intervention or percutaneous drainage was carried out. Also the hematoma was limited in the intraparenchymal or subcapsular region in those cases.
This case is the first and single one in the literature in terms of retroperitoneal hematoma after RIRS. The patient had no comorbidity and there was no use of anticoagulants. This hematoma did not transform into abscess, no surgical intervention was attempted, and the patient was treated by close follow-up and conservative treatment. The main indications of interventions like selective arterial embolization or surgery to the retroperitoneal hematomas include, abdominal compartment syndrome, and impaired cardiovascular or respiratory functions and instability of hemoglobine levels. 8 In this case, the massive retroperitoneal hematoma detected by CCT strongly implied staying in a stable condition and no additional venture was planned. Although, we have not made use of ultrasonography in the diagnosis and follow-up, it must be kept in mind that it is a less hazardous, easily available tool, especially if performed by the same operators, for detection and follow-up of this complication.

RIRS is a suitable and efficient option in the treatment of kidney stones with high success rate and acceptable complication rate. Retroperitoneal hematoma is a very rare and serious complication, which should be kept in mind but to date no case has been reported to our knowledge. In order to decrease the risk of perforation and bleeding, overflexion or deflexion of the flexible renoscope should be avoided in the lower pole calculi; particularly if stone burden is high, intrarenal pressure should be kept low by using UAS, and operational time should be preferably under an hour.

Once retroperitoneal hematoma is diagnosed, the patient should be treated immediately and cautiously as lack of treatment may lead to mortality. Management of retroperitoneal hematomas is a complex and conservative approach; while being closely monitored, might be an option, especially if the patient is hemodynamically stable. The evidence from the literature revealed that fluid replacement, blood transfusion (if required), advising bed rest, and serial imaging follow-up are adequate in most cases of retroperitoneal hematomas. This case underscores a 
rare complication of a recent endourological procedure and highlights successful conservative management strategies.

\section{REFERENCES}

1. Turk C. Urolithiasis guidelines: Retrospective view and perspectives. Urologe A 2016; 55:1317-20.

2. de la Rosette J, Denstedt J, Geavlete P, Keeley F, Matsuda T, Pearle $\mathrm{M}$, et al. The clinical research office of the endourological society ureteroscopy global study: Indications, complications, and outcomes in 11,885 patients. J Endourol 2013; 28:131-9.

3. Bas O, Tuygun C, Dede O, Sari S, Çakici MÇ, Öztürk U, et al. Factors affecting complication rates of retrograde flexible ureterorenoscopy: Analysis of 1,571 procedures - a singlecenter experience. World J Urol 2017; 35:819-26.

4. Chen S, Xu B, Liu N, Jiang H, Zhang X, Yang Y, et al. Improved effectiveness and safety of flexible ureteroscopy for renal calculi $(<2 \mathrm{~cm})$ : A retrospective study. Can Urol Assoc J 2015; 9:E273-7.
5. Watanabe R, Inada K, Azuma K, Yamashita Y, Oka A. Case of renal subcapsular hematoma caused by flexible transurethral lithotripsy. Hinyokika Kiyo 2013; 59:565-8.

6. Paiva MM, da Silva RD, Jaworski P, Kim FJ, Molina WR. Subcapsular hematoma after ureteroscopy and laser litotripsy. Can J Urol 2016; 23:8385-7.

7. Sbrana F, Pasanisi EM. A massive retroperitoneal hematoma during low-molecular weight-heparin therapy. Intern Emerg Med 2016; 11:153-4.

8. Sunga KL, Bellolio MF, Glimore RM, Cabrera D. Spontaneous retroperitoneal hematoma; etiology, caracteristics, management, and outcome. J Emerg Med 2012; 43:157-61.

9. Yahsi S, Tonyali S, Ceylan C, Yildiz KY, Ozdal L. Intraparenchymal hematoma as a late complication of retrograde intrarenal surgery. Int Braz J Urol 2017; 43:367-70.

10. Salvadó JA, Consigliere L, Gallegos H, Rojas F, Astroza G. Subcapsular renal-infected hematoma after retrograde intrarenal surgery: A rare but serious complication. J Endourol Case Rep 2016; 2:52-4

.......... 